\title{
Filterkuchenwäsche zur Aufreinigung löslicher kristalliner Feststoffpartikel
}

\section{Franky Ruslim*, Jenny Fleischer, Hermann Nirschl und Urs Peuker}

Die Filterkuchenwäsche ist ein weit verbreitetes Verfahren zur Aufreinigung partikulärer Feststoffsysteme in Flüssigkeiten. Die Anwendung dieses Verfahrens auf lösliche kristalline Produkte hat allerdings oft einen höheren Waschflüssigkeitsverbrauch sowie einen Produktverlust zu Folge.

\section{Problemstellung}

Zahlreiche Produkte in der chemischen, Lebensmittel-, und Pharmaindustrie müssen zunehmend verschärfte Anforderungen hinsichtlich ihrer Reinheit erfüllen. Daher wird oft ein zusätzlicher Aufreinigungsschritt im und/oder nach dem Herstellprozess eingesetzt. Ein weit verbreiteter Aufreinigungsprozess ist die Auswaschung von Verunreinigung aus einem Fest/Flüssig-System durch Zugabe von einer sauberen Waschflüssigkeit.

Es sind zwei Grundwaschverfahren bekannt: die Durchströmungswäsche von Filterkuchen und die Verdünnungswäsche. Die Filterkuchenwäsche wird oft als Zwischenstufe in einer Verfahrenskette eingesetzt, bei der nach der Kuchenbildung die Waschflüssigkeit direkt auf die Oberfläche des Kuchens gegeben wird. Durch Anlegen eines treibenden Potenzials, z. B. Druckdifferenz oder Massenkräfte, dringt die Waschflüssigkeit in das Porensystem des Filterkuchens ein und verdrängt die Mutterflüssigkeit samt Verunreinigung. Bei der Verdünnungswäsche wird das Feststoffhaufwerk in einer Waschflüssigkeit angemaischt, und dies führt zu einer Auflösung der Haufwerkstruktur. Es kommt zu einer Vermischung von Mutter- und Waschflüssigkeit, wodurch die Diffusionswege im Vergleich zur Filterkuchenwäsche erheblich verkürzt werden und die Konzentration der $\mathrm{zu}$ entfernenden gelösten Substanz herabgesetzt wird. Um hohe Aufreinigungsgrade $\mathrm{zu}$ erreichen, wird die Verdünnungswäsche oft mehrstufig betrieben. Hierfür sind mehrere Trenn- und Resuspendierungsschritte notwendig.

Ein Großteil der Feststoffprodukte, die aufgereinigt werden müssen, wird in kristalliner Form gewonnen und ist daher in vielen Fällen auch in der Waschflüssigkeit löslich. Aufgrund der Lösungsproblematik stellt die effektive Waschung löslicher Produkte eine besondere
Herausforderung dar. Ein Anwendungsgebiet ist z. B. die Waschung von Kalium-Ionen aus Natriumchlorid (Kochsalz).

\section{Grundlagen}

Ist das Feststoffprodukt sehr gut löslich in der Waschflüssigkeit, so ist eine Verdünnungswäsche kaum möglich, da die Waschflüssigkeit den Feststoff stark erodiert. Bei einer schnellen Lösungskinetik sättigt sich die Waschflüssigkeit nahezu vollständig mit dem Produkt, was zu einem Ausbeuteverlust führt.

Die Durchströmungswäsche nutzt die Kapillarstruktur des Filterkuchens zu einer mehr oder weniger kolbenförmigen Verdrängung der Mutterflüssigkeit. Durch die auftretenden Dispersions- und Diffusionsvorgänge in der Flüssigkeit sowie Ad- und Desorptionsvorgänge an den Partikeloberflächen ist allerdings ein vollständiger Flüssigkeitsaustausch nicht realisierbar. Bei der technischen Realisierung von Waschverfahren muss daher zwischen Aufwand und Aufreinigungsergebnis abgewogen werden.

Zur Darstellung der Waschergebnisse wird in der Regel der Restverunreinigungsanteil $X^{* *}$ als Funktion des Waschverhältnisses aufgetragen (s. Abb. 1). Der Restverunreinigungsanteil ist definiert als die Restmassenbeladung der Verunreinigung bezogen auf die Anfangsbeladung $X_{0}$. Bei konstant bleibender Produktmasse ist es gleichzeitig das Verhältnis der Verunreinigungsmasse nach und vor dem Waschprozess (Gl. (1)). Bei der Auswertung nach $X^{*}$ ist es unerheblich, ob die Reinigung durch den Waschvorgang oder eine Entfeuchtung stattgefunden hat.

$X^{*}=\frac{X}{X_{0}}=\frac{m_{\mathrm{V}}}{m_{\mathrm{V}, 0}}$

\section{Ein weit verbreite- ter Aufreinigungs- prozess ist die Auswaschung von Verunreinigung aus einem Fest/Flüssig- System durch Zu- gabe von einer sauberen Wasch- flüssigkeit.}

\section{Ist das Feststoff- produkt sehr gut löslich in der Wasch- flüssigkeit, so ist eine Verdünnungs- wäsche kaum mög- lich, da die Wasch- flüssigkeit den Fest- stoff stark erodiert.}




\section{Die Fälle der idealen Verdünnung und der idealen Verdrän- gung stellen Grenz- fälle dar, zwischen denen sich die meisten Waschver- fahren der Kuchen- wäsche bewegen.}

Das Waschverhältnis gibt das Waschflüssigkeitsvolumen $V_{\mathrm{W}}$ als Vielfaches des Porenvolumens $V_{\mathrm{H}}$ an (Gl. (2)):

$\mathrm{W}=\frac{V_{\mathrm{W}}}{V_{\mathrm{H}}}$

Ein Waschverhältnis von $W=1$ entspricht einem einfachen Hohlraumvolumen, somit theoretisch der Menge an Waschflüssigkeit, die benötigt wird, um unter Voraussetzung einer idealen Verdrängung bei einer Sättigung von $S=1$ des Haufwerks das gesamte Porenvolumen auszutauschen.

Die Auftragung des Restverunreinigungsanteils als Funktion des Waschverhältnisses ergibt eine modifizierte Waschkurve (s. Abb. 1). Diese Waschkurve enthält sowohl die Reinigung durch eine Entfeuchtung des Kapillarsystems als auch durch die eigentliche Waschung mit der Waschflüssigkeit. Aus diesem Grund schneidet die Waschkurve die Ordinate nicht wie bei einer konventionellen Waschkurve bei $X^{*}$ gleich Eins, sondern bei einem Wert kleiner Eins, der vom erreichten Entfeuchtungszustand abhängt.

Die Fälle der idealen Verdünnung und der idealen Verdrängung stellen Grenzfälle dar, zwischen denen sich die meisten Waschverfahren der Kuchenwäsche bewegen. Die Waschkurve wird in drei charakteristische Bereiche unterteilt, in denen unterschiedliche Stofftransportmechanismen dominieren (s. Abb. 1).

Im Verdrängungsbereich (Bereich I) wird die Porenflüssigkeit durch die Waschflüssigkeit nahezu kolbenförmig verdrängt. Sind die größeren Poren bereits von reiner Waschflüssigkeit durchströmt, so laufen verschiedene Stofftransportmechanismen wie Konvektion, Dispersion und Diffusion parallel ab (Bereich

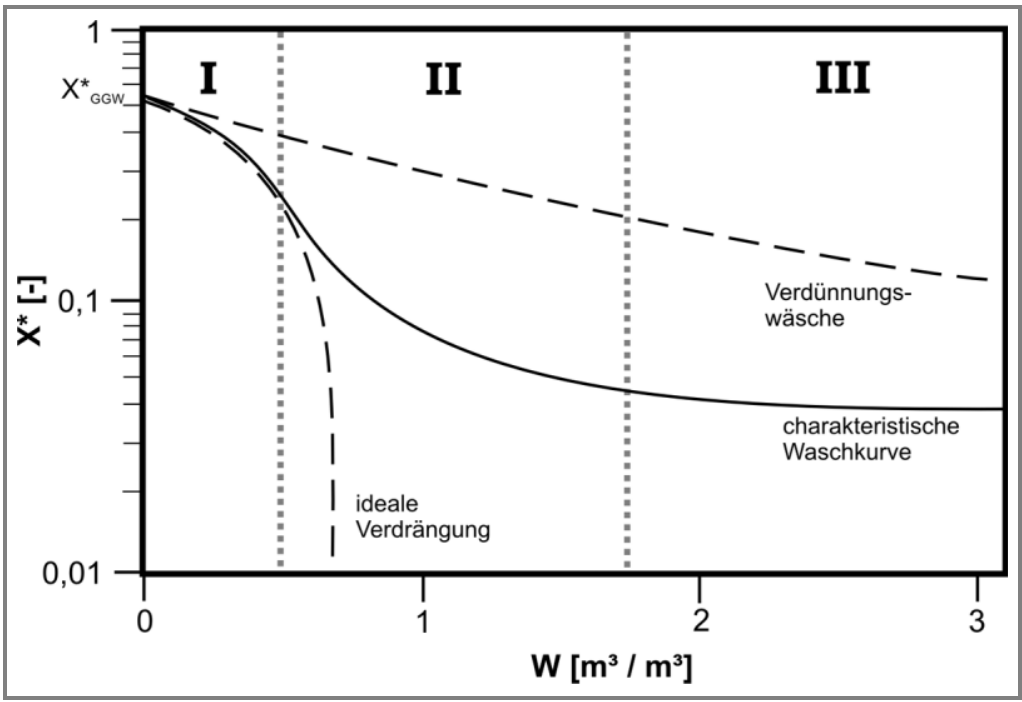

Abbildung 1. Typischer Verlauf einer Waschkurve unter Berücksichtigung des Aufreinigungseffektes durch die Entfeuchtung.
II). Bei der Dispersion handelt es sich um strömungsinduzierte Mischungsvorgänge der Flüssigkeiten, die eine ideale Verdrängung der Mutterflüssigkeit durch die Waschflüssigkeit verhindern. Im Diffusionsbereich (Bereich III) sind die durchströmbaren Kapillaren von den verdrängbaren Anteilen der Mutterflüssigkeit befreit. Verunreinigungen werden ausschließlich durch Diffusion aus den stagnierenden Bereichen des Filterkuchens in die Waschflüssigkeit transportiert [1].

\section{Messprinzip und Versuchs- aufbau}

Als Modellstoffsystem für die Untersuchung wurde das System Natriumchloridsalz/demineralisiertes Wasser/Fluorescein verwendet. $\mathrm{NaCl}$ ist das kristalline Feststoffprodukt, das künstlich mit dem Farbstoff Fluorescein (auch bekannt aus der Fluoreszenzspektroskopie) verunreinigt wird. Die Löslichkeit von $\mathrm{NaCl}$ in demineralisiertem Wasser beträgt $358 \mathrm{~g} / \mathrm{kg}$. Die Mutterlauge oder Porenflüssigkeit besteht aus gesättigter $\mathrm{NaCl}$-Lösung und der Verunreinigung. Zur Bestimmung der Verunreinigungskonzentration wird ein Photometer eingesetzt.

Einer der Schwerpunkte dieser Arbeit ist ein Apparate-Screening zur Untersuchung der Durchführbarkeit des Waschverfahrens zur Aufreinigung löslicher Produkte. Dabei werden mehrere Apparate eingesetzt: eine Waschsäule im Erdschwerefeld, eine Drucknutsche und ein Sedimentator [2].

Die Waschsäule besteht aus einer aus Plexiglas angefertigten Kuchenbildungseinheit, einer Tuchauflage mit Lochblech und einem Filtratablaufkanal. Die Kuchenhöhe ist durch den Einsatz verschiedener Kuchenbildungseinheiten unterschiedlicher Höhen variierbar. Bei einer zu geringen Kuchenhöhe überwiegen Randeffekte, und der Filtermediumwiderstand gewinnt an Einfluss [3]. Bei einem zu hohen Kuchen erhöht sich der Produktverbrauch, der Kuchenwiderstand nimmt zu, und die Kinetik verlangsamt sich.

Die Waschflüssigkeitsaufgabe stellt bei der Waschung von löslichen Produkten eine Herausforderung dar, da die oberste Kuchenschicht beim Auftreffen der Waschflüssigkeit angelöst und oder aufgewirbelt wird und dies $\mathrm{zu}$ einer bevorzugten Durchströmung der Waschflüssigkeit führt. Um das Aufwirbeln des Feststoffs zu verringern, kann ein Filtertuch bzw. eine Aufgabehilfe verwendet werden, die den Filterkuchen schützt und gleichzeitig die Waschflüssigkeit gleichmäßig auf der Kuchenoberfläche verteilt. 
Um Produktverlust durch den Lösevorgang während der Waschung zu vermeiden, wurde mit einer produktgesättigten Waschflüssigkeit gewaschen. Dies bedeutet für eine Wirtschaftlichkeitsbetrachtung, dass zur Herstellung dieser Waschflüssigkeit wiederum Produkt verbraucht wird. Um diesen Aufwand als entscheidenden Parameter zu berücksichtigen, führt man den Salzverbrauch (SV) ein, der gleichzeitig proportional zur zugegebenen Waschflüssigkeitsmenge bzw. Waschverhältnis W ist (Gl. (3)):

$$
\begin{aligned}
\mathrm{SV} & =\frac{m_{\mathrm{NaCl} \text { in der Waschflüssigkeit }}}{m_{\mathrm{P}}} \\
& =\frac{W V_{\mathrm{H}} \frac{358}{1358} \rho_{\mathrm{Lsg}}}{m_{\mathrm{P}}}
\end{aligned}
$$

\section{Experimentelle Ergebnisse}

Die modifizierte Waschkurve für die Waschsäule (s. Abb. 2) zeigt einen für die Haufwerkswäsche charakteristischen Verlauf. Dabei wird kein großes Potenzial zur Entfeuchtung aufgebracht, und das Kapillarsystem des Kuchens bleibt somit gefüllt. Die drei Bereiche ideale Verdrängung, Dispersion und Diffusion sind deutlich erkennbar. Der Diffusionsbereich wird bei einem Waschverhältnis von 1,5 erreicht. Es ist kein signifikanter Einfluss der Wahl des Filtermediums zu beobachten.

In der Regel wird die optimale Maschenweite und Beschaffenheit des Filtertuches durch die Filtrationseigenschaften der Suspension ermittelt, wobei die Partikelgröße eine bestimmende Größe ist. Aufgrund der Löslichkeit des Produktes kann es zu einer Verringerung der Partikelgröße während des Waschprozesses kommen, wenn die Oberfläche der Partikel angelöst wird. Dies wird durch die Verwendung von mit Produkt gesättigter Waschflüssigkeit vermieden. Dennoch lässt sich eine Abnahme der Partikelgröße vor und nach der Waschung feststellen (s. Abb. 3). Der Partikelgrößenverteilung ist $\mathrm{zu}$ entnehmen, dass der mittlere Partikeldurchmesser $d_{50}$ von $477 \mu \mathrm{m}$ auf $446 \mu \mathrm{m}$ abnimmt.

Wird das treibende Potenzial zur Entfeuchtung erhöht, beispielsweise in einer Nutsche (Überdruck) oder durch eine zusätzliche hydrostatische Druckdifferenz, so kann der Kuchen vor, während oder nach der Waschung untersättigt werden. Der Einfluss des treibenden Potenzials ist aus Abb. 4 ersichtlich. Durch Erhöhung des Potenzials $\Delta p$ in der Drucknutsche (hier von 5 auf 70 mbar Druckdifferenz) erfolgt die Aufreinigung schon bei niedrigeren Waschverhältnissen im Vergleich zu den vorherigen Ergebnissen. Die Erhöhung

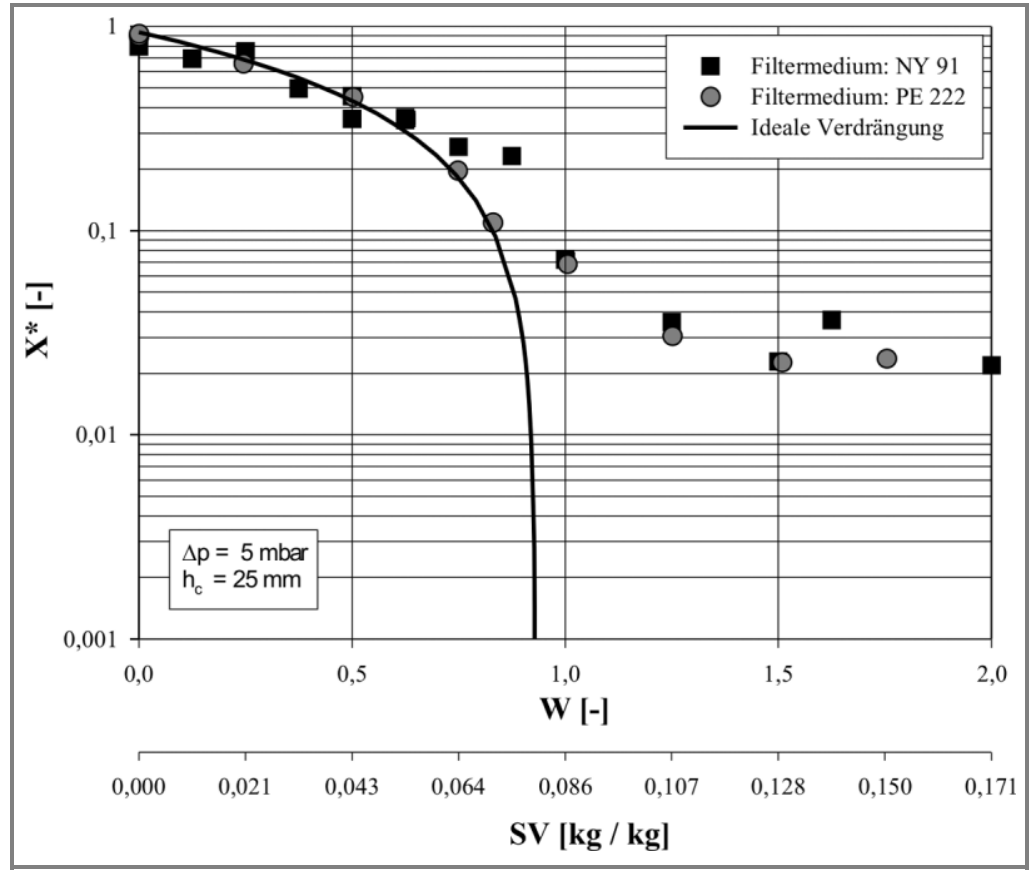

Abbildung 2. Ergebnisse der Filterkuchenwäsche in der Waschsäule bei der Variation der Filtermedien.

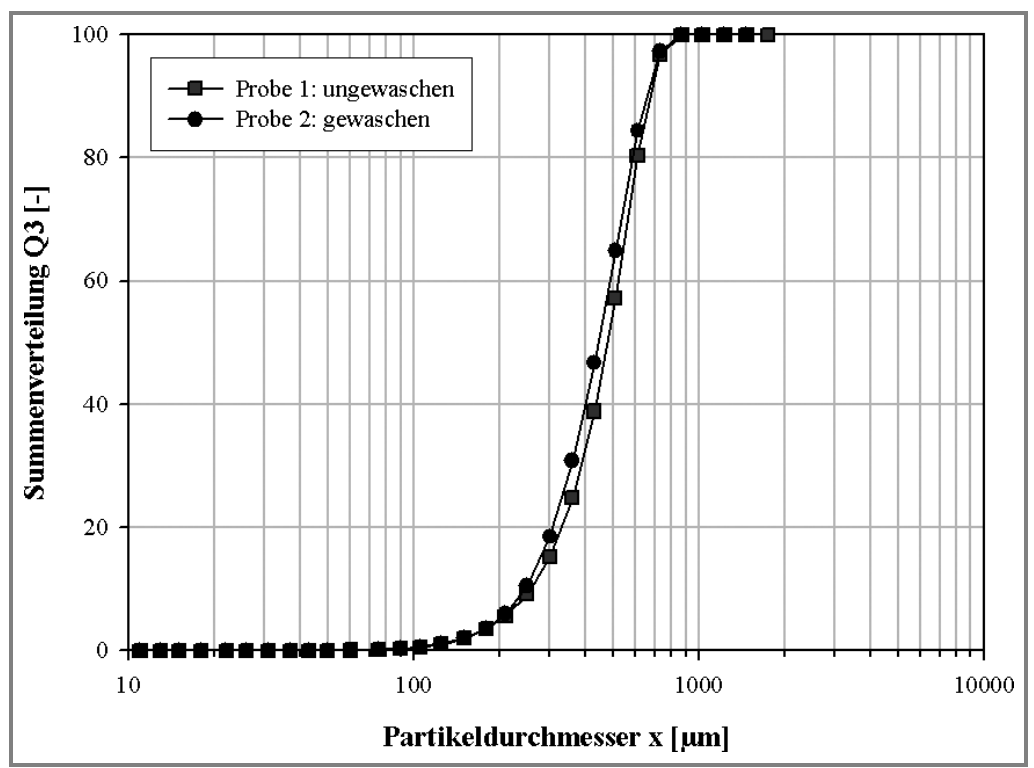

Abbildung 3. Partikelgrößenverteilung der ungewaschenen sowie gewaschenen $\mathrm{NaCl}$-Probe.

des treibenden Potenzials verbessert die Entfeuchtung und damit auch die Reinigung. Die Ergebnisse bei 70 mbar sind in den ersten beiden Bereichen der Waschkurve parallel zu geringeren $X^{*}$-Werten hin verschoben. Die Restverunreinigungen laufen im Diffusionsbereich wieder näher zusammen. Dies deutet auf eine konvektionskontrollierte Aufreinigung hin. Die Verunreinigung wird aufgrund der höheren Strömungskräfte besser verdrängt und ausgewaschen. 


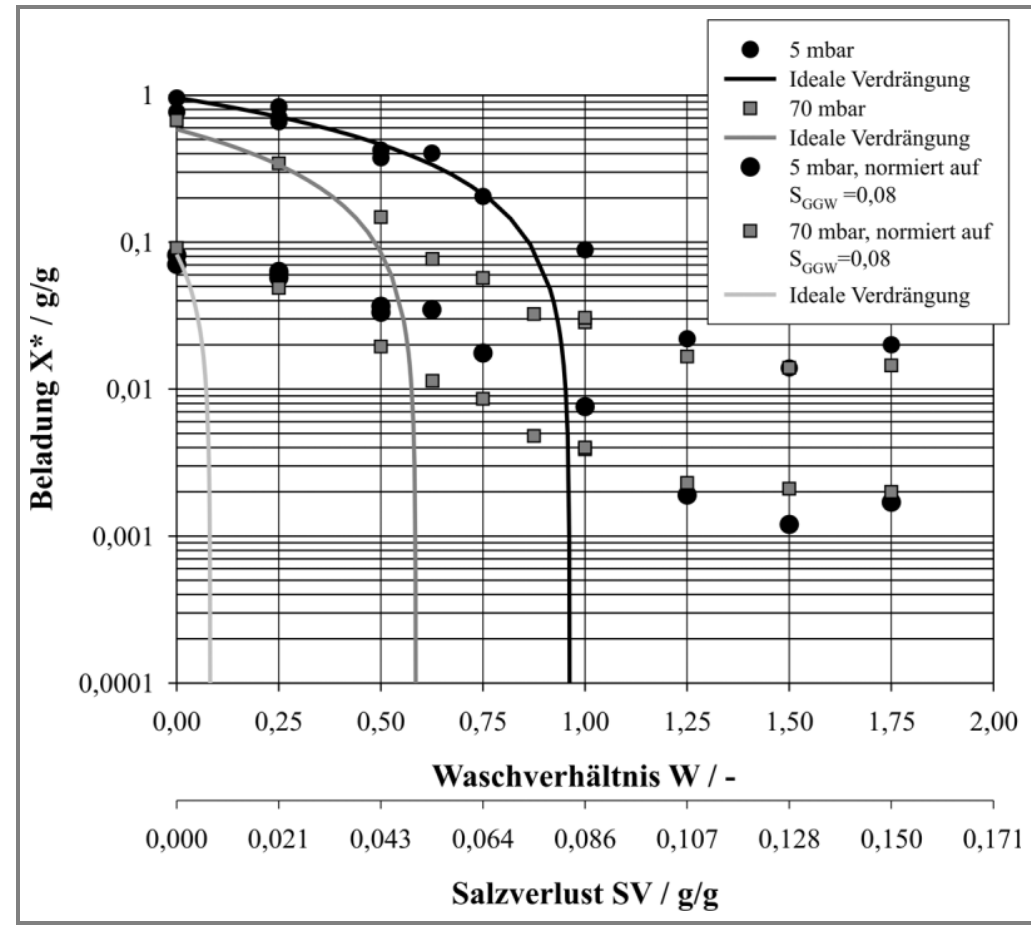

Abbildung 4. Einfluss des treibenden Potenzials auf die Filterkuchenwäsche.

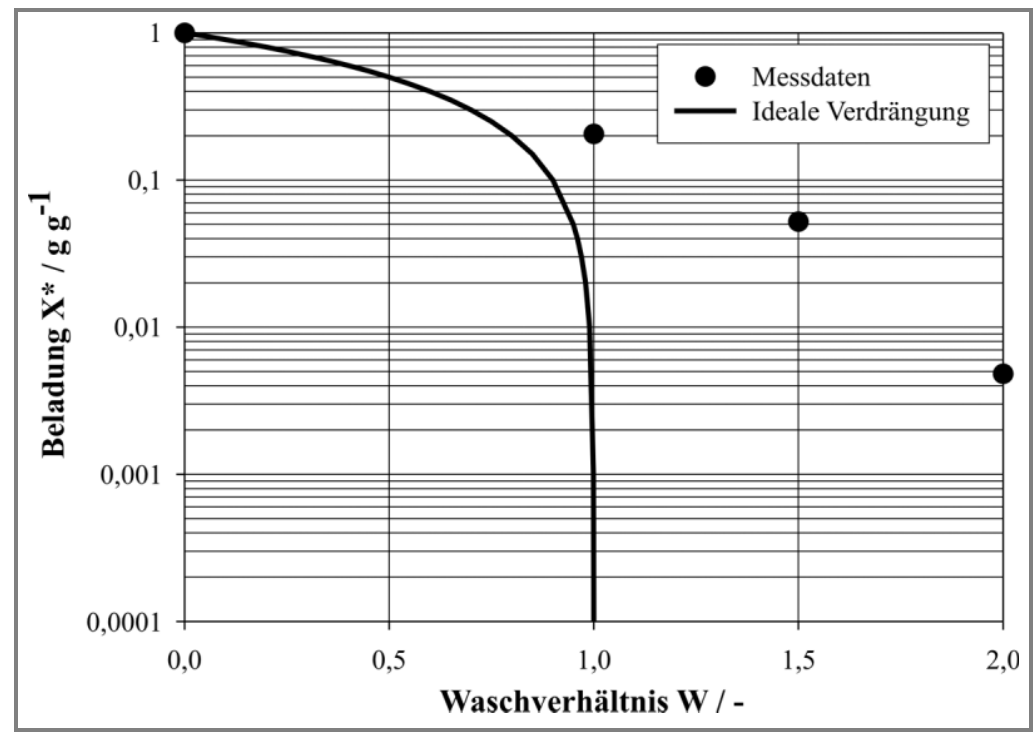

Abbildung 5. Ergebnisse der Wanderbettwäsche im Sedimentator.

Der Einfluss der Entfeuchtung auf den Aufreinigungsvorgang ist in einer Zentrifuge durch die erreichbaren geringen Sättigungen am Bond-Plateau nochmals deutlich erhöht. Aus den Waschergebnissen in der Waschsäule wird auf eine filtrierende Zentrifuge extrapoliert. Deshalb sind die Werte auf die entsprechenden Restverunreinigungsanteile bei einer Gleichgewichtsättigung $S=0,08$, die in einer Zentrifuge [4] mechanisch realisierbar ist, umgerechnet. Bei den berechneten Werten handelt es sich allerdings um eine Abschätzung unter der Annahme, dass die Verunreinigung gleichmäßig verteilt in der im Kuchen erhaltenen Flüssigkeitsmenge vorliegt und mit einer Entfeuchtung entsprechend dem Sättigungsverhältnis verringert werden kann. Die tatsächlichen Werte würden aufgrund von Inhomogenitäten und stagnierenden Bereichen höher liegen.

Das Waschverfahren im Sedimentator basiert auf einem durch Massenkräfte vertikal nach unten strömenden Bett, dem Wanderbett, das in einer Waschkammer horizontal von Waschflüssigkeit durchströmt wird [2]. Die Partikel bewegen sich relativ zueinander, und dies führt zur Vermeidung der stagnierenden Bereiche. Die Struktur des Feststoffverbandes wird vergleichmäßigt, und alle Partikel und partikelnahen Bereiche werden im Verlauf der Waschung aufgereinigt. Dieser Prozess wird daher als Einzelkornwaschung bezeichnet [5].

Die Ergebnisse zur Waschung von $\mathrm{NaCl}$ Kristallen im Sedimentator sind in Abb. 5 dargestellt. Daraus ist ersichtlich, dass die Asymptote $\left(X^{*} \sim 0,02 \mathrm{im}\right.$ Diffusionsbereich), die bei der Filterkuchenwäsche in der Waschsäule und Drucknutsche beobachtet wurde, hier bereits bei einem Waschverhältnis von ca. 1,7 unterschritten wird. Der Verlauf der Kurve zeigt, dass der Aufreinigungseffekt bis hin zu niedrigeren Verunreinigungsanteilen noch möglich ist. Hier macht sich der Vorteil der Wanderbettwäsche bemerkbar, dass stagnierende Bereiche, die im Festbett vorkommen, der Waschflüssigkeit zugänglich gemacht werden. Da die Variation der Wachverhältnisse über den Waschflüssigkeitsvolumenstrom geregelt wird und sich dieser mit der Wandergeschwindigkeit des Feststoffbettes erhöht, ist es nicht möglich höhere Waschverhältnisse einzustellen, ohne dass Feststoffpartikel von der Flüssigkeitsstrom mitgerissen und ausgetragen werden. Da die Porosität im Wanderbett bei $\varepsilon=0,42$ und nicht bei 0,37 wie im Festbett liegt, ist bei gleichem Waschverhältnis der Waschflüssigkeitsbedarf und damit auch der Salzverbrauch höher.

Ein Vergleich des Salzverbrauches für die beiden Verfahren ist in Abb. 6 dargestellt.

\section{Zusammenfassung und Bedeutung für die Praxis}

Ein Großteil der in der Verfahrenstechnik verarbeiteten Produkte sind kristalline Feststoffpartikel. Aufgrund der Löslichkeit dieser Feststoffe sowohl in der Mutterlauge als auch in der Waschflüssigkeit ist die Waschung von solchen Produkten sehr problematisch. Bei hohen Löslichkeiten und schneller Löslich- 
keitskinetik des Produktes wie bei $\mathrm{NaCl}$ und demineralisiertem Wasser kommt die Verdünnungswaschung aufgrund des hohen Produktverlustes nicht in Frage. Eine Verringerung der Kontaktzeit zwischen den Fest/FlüssigPhasen ist bei der Filterkuchenwäsche durch Verwendung hoher Strömungspotenziale wie beispielsweise Druck- oder Massenkraft möglich. Um das Problem der Löslichkeit zu umgehen, wird mit einer gesättigten Lösung gewaschen, und dies bedeutet gleichzeitig einen Produktverbrauch zur Herstellung der Waschflüssigkeit. Der Produktverbrauch für verschiedene Waschverfahren wurde verglichen.

Die Waschung nach dem Wanderbettverfahren im Sedimentator ermöglicht eine weitergehende Verringerung des Verunreinigungsanteils. Im Gegensatz dazu ist keine weitere Abnahme des Verunreinigungsanteils ab einem Waschverhältnis von 1,25 bei der Waschung im Erdschwerefeld möglich, da stagnierende Bereiche nicht mehr durchströmt werden können (s. Abb. 4). Eine Erhöhung des treibenden Potenzials durch eine hydraulische Druckdifferenz führt zwar zur effizienteren Waschung schon bei niedrigen Waschverhältnissen, ändert allerdings das Niveau des erreichbaren Restverunreinigungsanteils nicht. Der Nachteil des Sedimentators ist der höhere Produktverbrauch zur Herstellung der Waschflüssigkeit und die Limitierung durch Partikelaustrag bei hohen Volumenströmen.

Eingegangen am 23. Januar 2006

\section{Formelzeichen}

$\begin{array}{lll}\varepsilon & {[-]} & \text { Porosität } \\ \rho & {\left[\mathrm{kg} / \mathrm{m}^{3}\right]} & \text { Dichte } \\ m_{\mathrm{P}} & {[\mathrm{kg}]} & \text { Produktmasse } \\ m_{\mathrm{V}} & {[\mathrm{kg}]} & \text { Verunreinigungsmasse } \\ m_{\mathrm{V}, 0} & {[\mathrm{~kg}]} & \text { Anfangsmasse } \\ \mathrm{SV} & {[\mathrm{kg} / \mathrm{kg}]} & \text { Salzverbrauch } \\ V_{\mathrm{H}} & {\left[\mathrm{m}^{3}\right]} & \text { Hohlraumvolumen } \\ V_{\mathrm{W}} & {\left[\mathrm{m}^{3}\right]} & \text { Waschflüssigkeitsvolumen } \\ \mathrm{W} & {[-]} & \text { Waschverhältnis } \\ X & {[\mathrm{~kg} / \mathrm{kg}]} & \text { Beladung } \\ X^{*} & {[-]} & \text { Restverunreinigungsanteil }\end{array}$

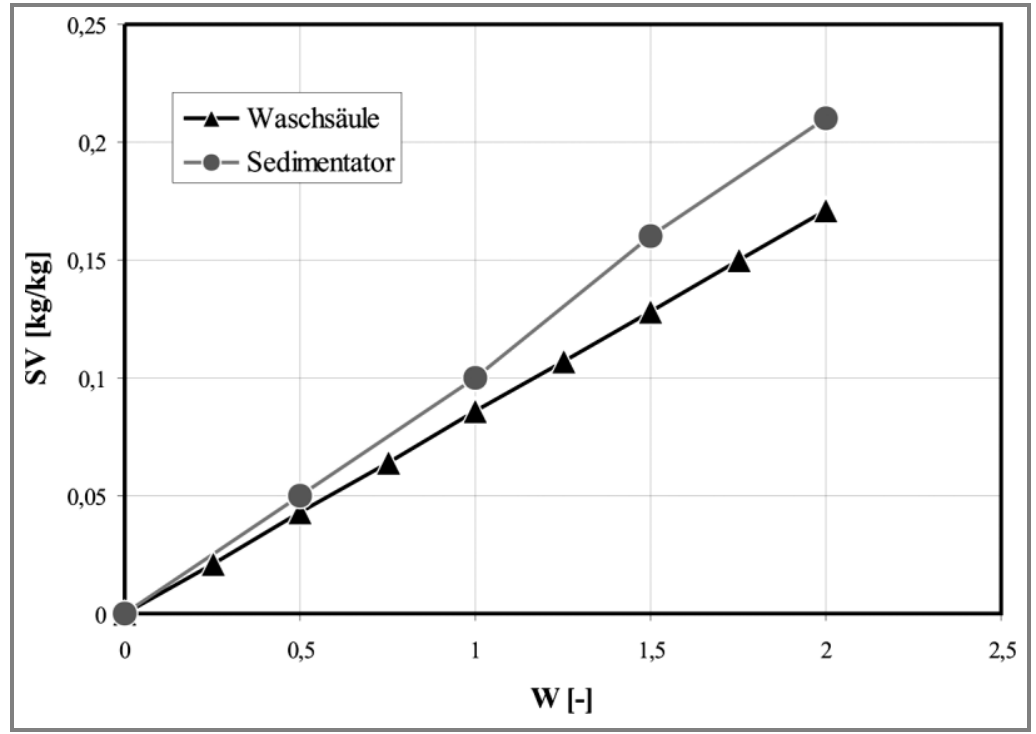

Abbildung 6. Vergleich des Salzverbrauches für die Filterkuchenwäsche in der Säule und im Sedimentator.

\section{F. Ruslim}

(franky.ruslim@mvm.uni-karlsruhe.de),

\section{J. Fleischer,}

\section{H. Nirschl,}

\section{U. Peuker,}

Universität Karlsruhe (TH), Institut für

Mechanische Verfahrenstechnik und Mechanik,

Kaiserstraße 12, D-76128 Karlsruhe, Germany.

\section{Literatur}

[1] W. Stahl, H. Anlauf, Kurs Fest/Flüssig-Trennung, Universität Karlsruhe (TH) 2003.

[2] B. Hoffner, W. Stahl, Chem. Ing. Tech. 2003 , 75 (8), 1173. DOI: 10.1002/cite.200390444

[3] J. Heuser, Dissertation, Universität Karlsruhe (TH) 2003.

[4] U. A. Peuker, Dissertation, Universität Karlsruhe (TH) 2002.

[5] B. Hoffner et al., Chem. Ing. Tech. 2004, 76 (9), 1417. DOI: $10.1002 /$ cite. 200490377

[6] B. F. Voitkovskii, A. V. Protsenko, Koks I Khimiya 1988, 2, 49.

\section{Eine Verringerung der Kontaktzeit zwischen den Fest/ Flüssig-Phasen ist bei der Filter- kuchenwäsche durch Verwendung hoher Strömungs- potenziale wie beispielsweise Druck- oder Mas- senkraft möglich.}

